\title{
Fonética y fonología de las consonantes oclusivas del guaymí hablado en Costa Rica ${ }^{1}$
}

\section{(Phonetics and Phonology of Stop Consonants in the Guaymi Spoken in Costa Rica)}

\author{
Melany Vega Chavarría
}

Universidad de Costa Rica, San Pedro Montes de Oca, Costa Rica

\begin{abstract}
RESUMEN
El estudio consiste en un análisis de las propiedades fonéticas y fonológicas de las series $/ \mathrm{t}-\mathrm{k} / \mathrm{y} / \mathrm{b}-\mathrm{d}-\mathrm{g} /$ del guaymí hablado en La Casona, Puntarenas, Costa Rica. El corpus se analizó con Speech Analyzer y se aplicó el método de Lisker y Abramson (1964) para obtener los valores del V.O.T. Los resultados señalan que la serie $/ \mathrm{t}-\mathrm{k} /$ no presenta variantes alofónicas sistemáticas, mientras que /b-d-g/ alterna con alófonos oclusivos, fricativos y aproximantes; la neutralización entre fonemas oclusivos no es frecuente; $y$, por último, el V.O.T. contribuye a la diferenciación entre oclusivas sordas y sonoras.
\end{abstract}

\begin{abstract}
The present work consists of an analysis of the phonetic and phonological properties of the sequences /t-k/ and /b-d-g/ from the Guaymi spoken in La Casona, Puntarenas, Costa Rica. The corpus was analyzed with Speech Analyzer and the Lisker and Abramson (1964) method was applied in order to obtain the values of the V.O.T. The results suggest that the sequence /t-k/ does not present systematic allophonic variations, while /b-d-g/ alternates with stop, fricative, and approximant allophones; neutralization

1 Recibido: 25 de setiembre de 2020; aceptado: 16 de marzo de 2021. En este artículo se detallan los resultados de un estudio preliminar presentado en el $V$ Congreso Internacional de la Asociación Centroamericana de Lingüistica (ACALING), el cual se llevó a cabo en agosto del 2019, en el Campus Omar Dengo de la Universidad Nacional de Costa Rica.

2 Programa de Maestría en Lingüística; https://orcid.org/0000-0003-4340-7292. Correo electrónico: melanyvega.ch@gmail.com
\end{abstract}


among stop phonemes is not frequent. Lastly, the V.O.T. contributes to the differentiation between voiceless and voiced stops.

Palabras clave: guaymí, ngäbere, fonética, fonología, V.O.T., chibcha Keywords: Guaymi, Ngäbere, phonetics, phonology, V.O.T., Chibcha

\section{Introducción}

Este es un análisis de las propiedades fonéticas (acústicas) y fonológicas de las series de consonantes oclusivas /t-k/ y /b-d-g/ de la variedad de la lengua guaymí, también conocida como 'ngäbere', hablada en Costa Rica. Su objetivo principal es presentar los datos más relevantes del estatus fonológico de las oclusivas de la lengua, tomando en consideración las descripciones fonológicas previas.

Se presenta a partir de la siguiente estructura: en primer lugar, se ofrece una breve descripción del guaymí, su caracterización genealógica, geográfica y grado de vitalidad actual. En segundo lugar, se revisa la documentación fonológica más destacada sobre la lengua, la cual se extiende desde el primer trabajo registrado en 1882 de Franco y Pinart ${ }^{3}$, hasta la obra más reciente, que corresponde a la Gramática de la lengua guaymí. Dialecto de Costa Rica: Una descripción urgente de Murillo ${ }^{4}$, de 2016. En este apartado, además, se realiza una comparación entre los planteamientos relativos al estatus de los fonemas oclusivos del guaymí de la variedad costarricense - tomando como fuente principal la ya mencionada gramática de Murillo_- y la serie oclusiva del guaymí de la variedad hablada en Panamá, descrita por Miguel Ángel Quesada Pacheco en 20085. En tercer lugar, en el apartado que se destina a los resultados se describen los procesos fonológicos que afectan a las oclusivas de la lengua, los cuales están relacionados, principalmente,

3 Blas José Franco y Alphonse Louis Pinart, Noticias de los indios del Departamento de Veragua, y vocabularios de las lenguas guaymi, norteño, sabanero y dorasque (San Francisco, CA: A. L. Bancroft, 1882).

4 José Manuel Murillo Miranda, Gramática de la lengua guaymí. Dialecto de Costa Rica: Una descripción urgente (Editorial Académica Española, 2016).

5 Miguel Ángel Quesada Pacheco, Gramática de la lengua guaymí (ngäbe) (Muenchen: Lincom Europa, 2008). 
con fenómenos de debilitamiento consonántico, elisión y asimilación; también se incluyen imágenes espectrográficas y en forma de onda que ilustran tales procesos acústicos. Por último, se presentan los datos obtenidos del análisis del Voice Onset Time (V.O.T) de ambas series de consonantes, y se detallan las características acústicas derivadas de los valores del V.O.T resultantes en cada una de las series.

\section{Metodología}

Los datos originales de audio empleados en esta investigación, así como la traducción ortográfica y morfemática interlineal, fueron facilitados por Murillo 6 . Las fuentes de audio consisten en grabaciones en formato mp3 de dos historias, las cuales fueron recolectadas gracias a la colaboración de dos hablantes de guaymí, pobladores de La Casona, Coto Brus, Provincia de Puntarenas, Costa Rica.

El procedimiento del trabajo fue el siguiente: se realizó el procesamiento del corpus audible con la ayuda del programa de análisis acústico de los sonidos del habla Speech Analyzer ${ }^{7}$, en su versión 3.1 , en grabaciones monoaurales con formato wav a $44.100 \mathrm{~Hz}$. Para cada uno de los audios se efectuó una transcripción fonética, basada en la percepción auditiva, y en el comportamiento de los sonidos que mostraron las imágenes del análisis acústico en forma de onda, espectrográfica y las líneas formánticas.

Tras el análisis acústico, con el fin de observar del comportamiento de los fonos oclusivos, se determinaron los contextos fonológicos de aparición tomando en cuenta sonidos contiguos, criterios relativos a la sílaba y a la acentuación. En primer lugar, se determinaron, por un lado, contextos fonológicos vocálicos del tipo: posición final total precedida de vocal $\left(\mathrm{V}_{-} \#\right)$, posición intervocálica $\left(\mathrm{V}_{-} \mathrm{V}\right)$, posición inicial total seguida de vocal (\#_V); por otro lado, contextos fonológicos

6 Comunicación personal, 4 de enero de 2019.

7 Summer Institute of Linguistics, Speech Analyzer: A Computer Program for Acoustic Analysis of Speech Sounds (versión 3.1), [Software de computación], (2018), <https://software.sil.org/ speech-analyzer/>. 
con presencia de consonante del tipo: precedida de consonante $\left(\mathrm{C}_{-} \mathrm{V}\right)$ y seguida de consonante $\left(V_{-}\right.$C). En segundo lugar, se consideró la posición de la oclusiva en la estructura silábica (coda o ataque), partiendo de la tendencia en guaymí hacia la sílaba abierta (CV). Por último, fue contemplada la acentuación (sílaba tónica, sílaba átona pretónica, sílaba átona postónica).

Con respecto al análisis del Voice Onset Time, para determinar su valor se midió en milisegundos (ms), con la ayuda de los cursores de tiempo, el periodo transcurrido entre la barra de explosión de los fonemas oclusivos y el inicio de la vibración de las cuerdas vocales de un total de 294 instancias de consonantes, en posición inicial absoluta y en medio de palabra. Se emplea el momento de la explosión como un cero referencial, de modo que, cuando la vibración de las cuerdas vocales inicia después de la barra de explosión, el valor del V.O.T. es positivo y, cuando inicia antes, negativo. Finalmente, se calculó el valor promedio del V.O.T de cada una de las consonantes oclusivas.

\section{Sobre la lengua}

El guaymí (exónimo) o ngäbere (endónimo) — también denominada ngöbe, ngäbere, ngäbe, novere, nove, movere ${ }^{8}$ — es una lengua de la familia chibcha, incluida en la subrama ístmica de la rama sureña, hermana del bocotá o buglere dentro del grupo guaymíico9 .

En Costa Rica el guaymí se habla en la zona del Pacífico Sur en los cantones de Buenos Aires, Golfito, Coto Brus y Corredores, en la provincia de Puntarenas, y cerca de la frontera con Panamá ${ }^{10}$. En el Censo Nacional de Población del 2011 elaborado por el Instituto Nacional de Estadística y Censos se estima que de un total de 3.654

8 Carlos Sánchez Avendaño, «Lenguas en peligro en Costa Rica: vitalidad, documentación y descripción», Káñina 37, 1 (2013): 219-250, 219.

9 Juan Diego Quesada, The Chibchan Languages (Cartago: Editorial Tecnológica de Costa Rica, 2007) 36.

10 Carlos Sánchez Avendaño, «Ngäbere/ ngäbe/ ngöbere/ novere/ nove/ guaymí», Diversidad y patrimonio lingüístico de Costa Rica. Universidad de Costa Rica, 26 de febrero de 2020, https://www. dipalicori.ucr.ac.cr/lengua-cultura/ngaberengoberenoverenoveguaymi/, §1. 
personas, unas 2.838 hablan la lengua, esto equivale a un $77,7 \%$ de la población residente en los territorios oficiales ${ }^{11}$. Sumado a esto, el análisis que efectúa Murillo de la lengua a partir de la clasificación baumaniana señala que, por el momento, la lengua no está gravemente amenazada y su grado de vitalidad puede calificarse como 'duradero' 12 . No obstante, Sánchez indica que, a pesar de que el guaymí es de las lenguas amerindias con mayor vitalidad en Costa Rica, también está experimentando un proceso de desplazamiento ${ }^{13}$.

\section{Antecedentes}

Las primeras labores de documentación sobre el guaymí son los trabajos de Franco y Pinart, y Alphonse ${ }^{14}$. Ambos son descripciones gramaticales que incluyeron un apartado con información sobre el inventario de sonidos de la lengua. La valía de estos primeros trabajos reside, sin duda, en su importancia histórica; sin embargo, la información que aportan resulta poco útil para una descripción actualizada. Durante el último cuarto del siglo pasado surgieron nuevos estudios, entre estos se encuentran Kopesec y Kopesec ${ }^{15}$, Lininger ${ }^{16}$, Arosemena $^{17}$ y Abarca ${ }^{18}$; los cuales corresponden a estudios sobre el inventario fonológico del guaymí. De forma más reciente, se encuentran la

11 Instituto Nacional de Estadística y Censos, Censo 2011. Población total en territorios indigenas por autoidentificación a la etnia indígena y habla de alguna lengua indigena, según pueblo y territorio indigena (2011), <https://www.inec.cr/documento/ censo-2011-poblacion-total-en-territorios-indigenas-por-autoidentificacion-la-etnia-0>.

12 Murillo, 15.

13 Sánchez (2013), 219.

14 Ephraim S. Alphonse, Guaymi Grammar and Dictionary: with Some Ethnological Notes (Washington, D.C.: U.S Government Printing Office, 1956).

15 Michael F. Kopesec y Bonnie M. Kopesec, «La jerarquía fonológica del guaymí», Lenguas de Panamá. Sistemas fonológicos (Panamá: Instituto Nacional de Cultura e Instituto Lingüístico de Verano, 1974) 17-30.

16 Bárbara Lininger Ross, «Estudios sobre el guaymí ngäbere: fonología, alfabeto y diccionario provisional», Revista de Filología y Lingüística 7, 1-2 (1981): 101-115.

17 Melquíades Arosemena, «Principales rasgos fonológicos del guaymí», Revista Latinoamericana de Estudios Etnolingüisticos 3 (1983): 87-119.

18 Rocío Abarca González, «Análisis fonológico del guaymí movere», Estudios de Lingüistica Chibcha 4 (1985): 7-46. 
Gramática de la lengua guaymí (ngäbe) de Miguel Ángel Quesada Pacheco, centrada en la variedad panameña de la lengua, y la Gramática de la lengua guaymí de Murillo, sobre el dialecto hablado en Costa Rica. Para efectos de esta investigación se han tomado como principales bases estos dos trabajos, justamente, por ser los que contienen información más actualizada. De las dos últimas fuentes citadas, se desprende que el sistema fonológico del guaymí cuenta con cinco fonemas oclusivos. La serie oclusiva no es simétrica, pues no cuenta con el fonema bilabial sordo /p/. Según señala Juan Diego Quesada, el bocotá, lengua hermana del guaymí, y otras lenguas que también pertenecen a la subrama ístmica chibchense como el boruca y el teribe, tampoco cuentan con el correlato oclusivo bilabial sordo en su repertorio ${ }^{19}$.

Algunos autores como Kopesec y Kopesec ${ }^{20}$ y Lininger ${ }^{21}$ han propuesto la existencia de fonemas oclusivos labializados en el guaymí; sin embargo, su existencia ha sido cuestionada debido a su escasa sistematicidad. Ante el desacuerdo, Murillo señala que incorporar una serie labializada complicaría el sistema de forma innecesaria, ya que no aporta nada diferente de uno cuya fonotáctica incluya la secuencia consonante-deslizante ${ }^{22}$. Acerca de las consonantes oclusivas de la variedad del guaymí de Costa Rica, Murillo propone lo siguiente:

1. Los fonemas oclusivos sordos dentoalveolar $/ \mathrm{t} / \mathrm{y}$ velar $/ \mathrm{k} / \mathrm{no}$ tienen variantes alofónicas ${ }^{23}$. Sin embargo, menciona casos de debilitamiento en entornos sonoros, ya sea intervocálicos o con presencia de consonantes nasales, de los que resultan las realizaciones oclusivas sonoras dentoalveolar [d] y velar [g] respectivamente ${ }^{24}$.

19 Juan Diego Quesada (2007), 51.

20 Kopesec y Kopesec, 25.

21 Lininger, 103.

22 Murillo, 25.

23 Murillo, 21.

24 Murillo, 32-33. 
2. Los fonemas oclusivos sonoros evidencian una alternancia entre alófonos oclusivos sonoros [b], [d], [g] y alófonos fricativos sonoros $[\beta],[ð]$ y $[\gamma]$; estos últimos se articulan en posición intervocálica ${ }^{25}$.

Por su parte, Miguel Ángel Quesada Pacheco afirma lo siguiente para la variedad de Panamá:

1. Los fonemas sordos $/ \mathrm{t} / \mathrm{y} / \mathrm{k} /$ tienen un solo alófono oclusivo sordo $[\mathrm{t}]$ y $[\mathrm{k}]$. Estos aparecen en posición prenuclear, es decir, a inicio de palabra o sílaba. Suelen alternar con un alófono oclusivo sonoro [d] y [g] en la conversación rápida y se sonorizan frente a vocal nasal, con lo cual, fonemas sordos y sonoros llegan a neutralizarse. La neutralización de los fonemas $/ \mathrm{t} / \mathrm{y}$ $/ \mathrm{d} / \mathrm{y} / \mathrm{k} / \mathrm{y} / \mathrm{g} /$ es un fenómeno tan frecuente que incluso se refleja en la escritura, pues los hablantes tienen dificultades para ser conscientes de su estatus fonológico ${ }^{26}$.

2. Los fonemas sonoros $/ \mathrm{b} /, / \mathrm{d} / \mathrm{y} / \mathrm{g} /$ tienen un solo alófono oclusivo sonoro [b], [d] y [g]. Aparecen en posición prenuclear. En la conversación rápida pueden sufrir debilitamiento y fricativizarse ${ }^{27}$.

En un marco más amplio, está el trabajo de tipo areal, tipológico y genético de Juan Diego Quesada sobre las lenguas que pertenecen a la familia chibcha. En Gramática del buglere $e^{28}$, lengua hermana del guaymí, este autor indica que en algunas lenguas de la familia chibcha es común que los fonemas obstruyentes sufran un conocido proceso de lenición consonántica ${ }^{29}$. En el caso del buglere, los fonemas oclusivos sordos, en especial el velar, se caracterizan por una tendencia al debilitamiento en entornos silábicos inacentuados, generalmente

25 Murillo, 21

26 Miguel Ángel Quesada Pacheco, 32.

27 Miguel Ángel Quesada Pacheco, 32.

28 Juan Diego Quesada, Gramática del buglere (Quito, Ecuador: Abya-Yala, 2012).

29 Juan Diego Quesada (2012), 50. 
intervocálicos, del cual resultan realizaciones sonoras o fricativas; en menor grado, estos fonemas también se sonorizan en sílabas tónicas siempre que estén precedidos de algún segmento silábico. Por su parte, los segmentos oclusivos sonoros tienden a la fricativizarse como resultado de la lenición en entornos con segmentos silábicos en sílaba inacentuada. En algunos casos, estos fonemas sufren un proceso total de elisión; sin embargo, el dentoalveolar presenta una ligera tendencia a mantenerse oclusivo ${ }^{30}$.

\section{Resultados}

\section{Caracterización fonética y distribución}

Los fonemas oclusivos sordos aparecen exclusivamente en posición inicial de sílaba, debido a la tendencia de sílaba abierta de tipo CV. El alófono oclusivo sordo se articula tanto en posición inicial de palabra como en posición intervocálica. A continuación, en el ejemplo 1 y el ejemplo 2 se ilustran estos entornos de aparición; en ellos se incluye la grafía, la transcripción fonética y su traducción:

Ejemplo 1. Articulación oclusiva del fonema /t/ a inicio de palabra y en posición intervocálica

$$
\begin{aligned}
& <\text { täen }>\text { ['tõ̃̃n] 'ver' } \\
& <\text { jutäte }>\text { [hu.'to.te] 'pueblo' }
\end{aligned}
$$

Ejemplo 2. Articulación oclusiva del fonema /k/ a inicio de palabra y en posición intervocálica

$<$ krire $>$ ['kri.re] 'apenas'

$<$ jakite $>$ [ha.'ki.te] 'practicar'

Los fonemas oclusivos sonoros también aparecen de forma exclusiva en posición inicial de sílaba. El alófono oclusivo sonoro

30 Juan Diego Quesada (2012), 36-37. 
se articula en posición inicial de palabra, pero, contrario a lo que sucede con las sordas, este alófono solo aparece de forma marginal en entornos sonoros, generalmente con presencia de consonantes líquida o nasal. En posición intervocálica aparecen, en su lugar, alófonos fricativos y en algunos casos aproximantes. El ejemplo 3, el ejemplo 4 y el ejemplo 5 muestran ambos entornos de aparición en cada una de las oclusivas:

Ejemplo 3. Articulación oclusiva del fonema /b/a inicio de palabra y caso marginal en entorno sonoro intervocálico

$$
\begin{aligned}
& <\text { bití> [bi.'tĩ] 'después' } \\
& <\text { sribi> [sri.'bi] 'trabajo' }
\end{aligned}
$$

Ejemplo 4. Articulación oclusiva del fonema /d/a inicio de palabra y caso marginal en entorno sonoro con presencia de consonante nasal y líquida

$$
\begin{aligned}
& <\text { dengä> ['dẽn.gp] 'sacar' } \\
& <\text { nändre }>\text { [ñ̃n.'dre] 'andar' }
\end{aligned}
$$

Ejemplo 5. Articulación oclusiva del fonema /g/a inicio de palabra y caso marginal en entorno sonoro con presencia de consonante nasal

$$
\begin{aligned}
& <\text { gre }>\text { [gre] 'veinte' } \\
& <\text { dengä }>\text { ['dẽy.gp] 'sacar' }
\end{aligned}
$$

\section{Procesos fonológicos}

En esta sección se exponen algunos fenómenos fonológicos que afectan a las oclusivas del guaymí, relacionados, principalmente, con procesos de debilitamiento consonántico, elisión y asimilación. Las figuras que se presentan para ilustrar los casos fueron extraídas 
del programa de procesamiento acústico Speech Analyzer; estas se grafican en dos tipos de análisis: forma de onda y espectrográfico.

\section{Debilitamiento}

Según Murillo, el debilitamiento consonántico en el guaymí de la variedad de Costa Rica tiende a ocurrir en entornos sonoros, ya sea completamente vocálicos o con la presencia de consonantes nasales ${ }^{31}$. En el análisis efectuado, se registraron pocos casos de debilitamiento de las oclusivas sordas; los pocos hallados aparecen, en efecto, en entornos sonoros, pero a diferencia de lo mencionado por este autor, no se registraron alternancias con sus correlatos sonoros [d] y [g].

Las oclusivas sordas $[\mathrm{t}]$ y $[\mathrm{k}]$ tienen características acústicas muy diferentes de, por ejemplo, las vocales, entendiendo que estas últimas corresponden a los sonidos en los que se produce una menor obstrucción en la salida del aire. Las oclusivas sordas no presentan formantes o armónicos al no tener vibración de las cuerdas vocales; más bien, se caracterizan por una acumulación progresiva de aire, sucedida por su violenta liberación. Este fue el comportamiento que mostró el análisis acústico de Speech Analyzer en la mayoría de los casos, en donde la oclusión se muestra como un blanco que representa la ausencia de onda periódica, seguida de una barra vertical de oclusión.

No obstante, se registraron casos aislados de debilitamiento de la oclusiva velar sorda $[\mathrm{k}]$ en entornos sonoros estrictamente vocálicos y en sílaba inacentuada, de los que resulta una realización fricativa velar sonora [y]. Uno de estos casos se ilustra en la figura 1; como se observa, hay una transición formántica continua.

31 Murillo, 32. 
Figura 1. Realización fricativa velar sonora $[x]$ del fonema /k/

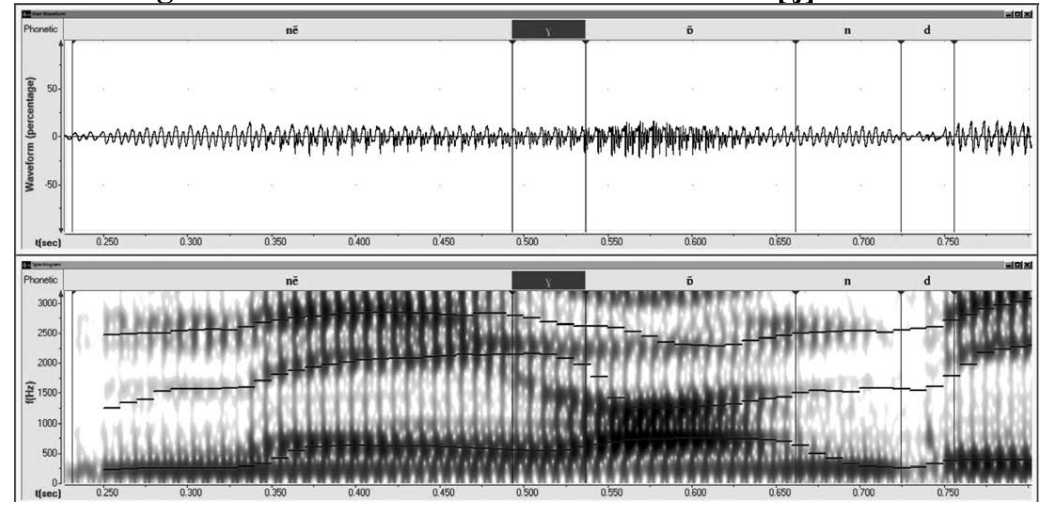

$<$ ne kändi> [nẽ.ỹ̃n.'di]

'DEM lugar'

Las consonantes oclusivas sonoras [b], [d] y [g] también presentan barra vertical de oclusión, pero se diferencian prototípicamente de las oclusivas sordas por mostrar una barra horizontal de sonoridad y por su duración más breve. Otros parámetros, como el V.O.T. (Voice Onset Time), también parecen ser determinantes en su distinción, razón por la cual más adelante se dedicará un apartado únicamente al análisis de los valores del V.O.T. de las oclusivas del guaymí. Se determinó que el debilitamiento de las oclusivas sonoras es bastante frecuente en entornos sonoros completamente vocálicos y, en menor medida, en entornos sonoros con presencia de consonantes sonoras. Esto da como resultado la aparición prácticamente sistemática de alófonos fricativos o aproximantes. En la figura 2 se ejemplifica la realización fricativa $[\beta]$ del fonema $/ b /$; como se muestra, no hay rastro alguno de barra de oclusión: 
Figura 2. Realización fricativa $[\beta]$ del fonema /b/ en posición intervocálica

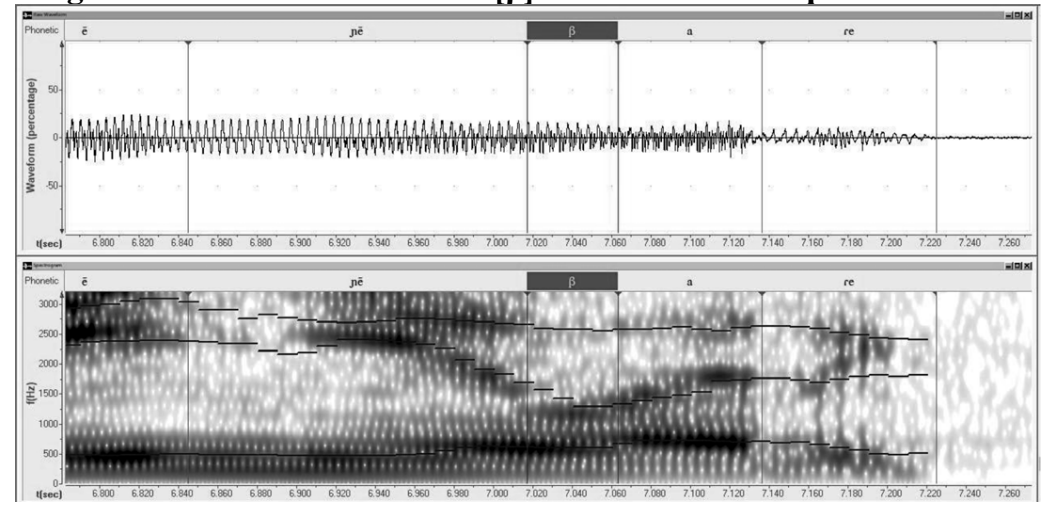

$<$ ñe-bare $>$ ['nẽ.ßßa.re]

'decir-PRF'

La figura 3 corresponde a una realización aproximante $[\beta]$ del fonema $/ \mathrm{b} /$. Los órganos articulatorios se aproximan sin establecer contacto. Si se compara la imagen espectrográfica de la aproximante con la de la fricativa anteriormente presentada, es posible notar una leve fluctuación en la altura de los formantes, así como el debilitamiento de formantes en el sector superior del espectro:

Figura 3. Realización aproximante $[\beta]$ del fonema /b/ en posición intervocálica

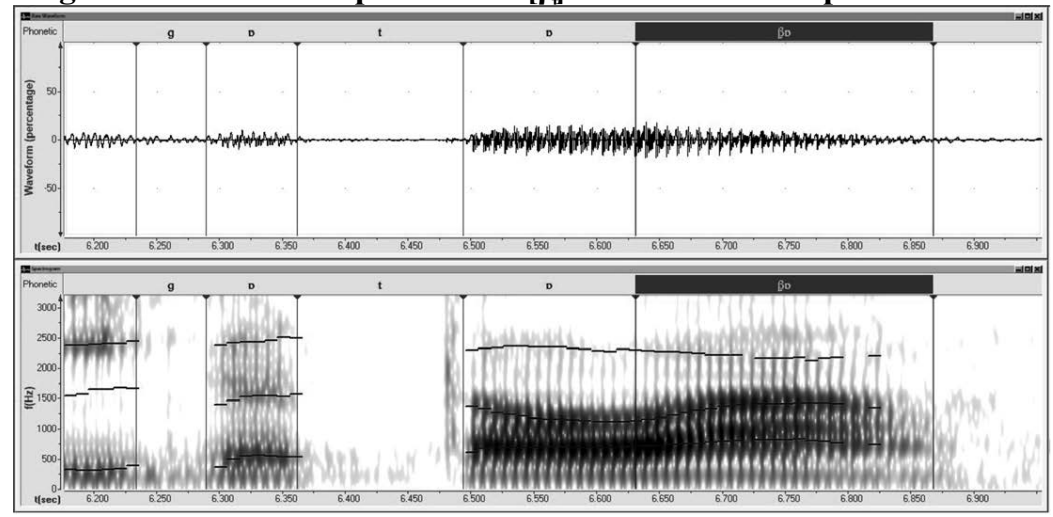

$<$ ngätä-ba $>$ [ti.gp.'to. $\beta$ p $]$

'encontrar-AS' 
En la figura 4 se ejemplifica la realización fricativa [ð] del fonema/d/. De la misma forma que con el fonema/b/, no hay rastro de barra vertical de oclusión y, por el contrario, se muestra una transición formántica continua:

Figura 4. Realización fricativa [ð] del fonema /d/ en posición intervocálica

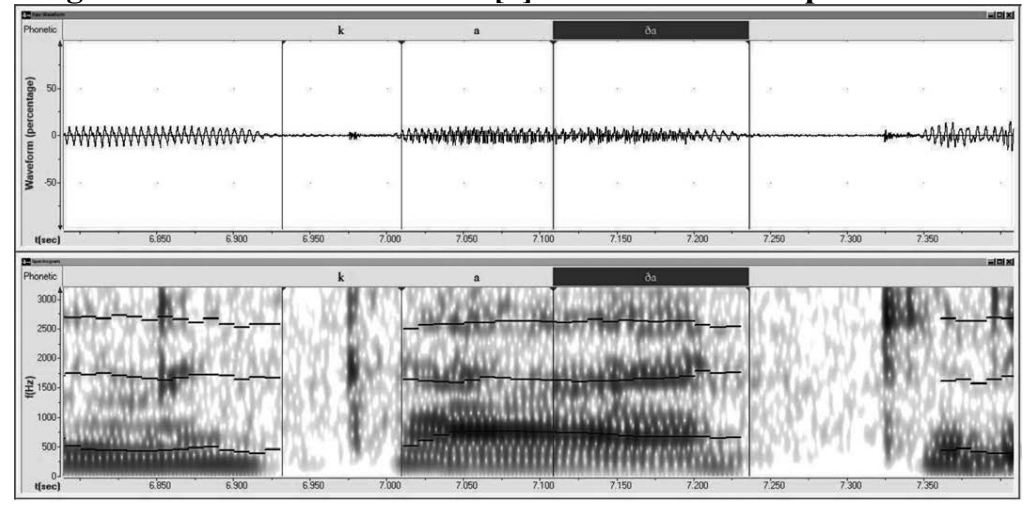

$<$ kada $>$ ['ka.ða]

'boca'

La realización aproximante [ð̛] del fonema /d/ se ilustra en la figura 5. Se detalla la fluctuación en la altura de los formantes, así como la semejanza formántica que guarda con la vocal contigua:

Figura 5. Realización aproximante [ọ] del fonema /d/ en posición intervocálica

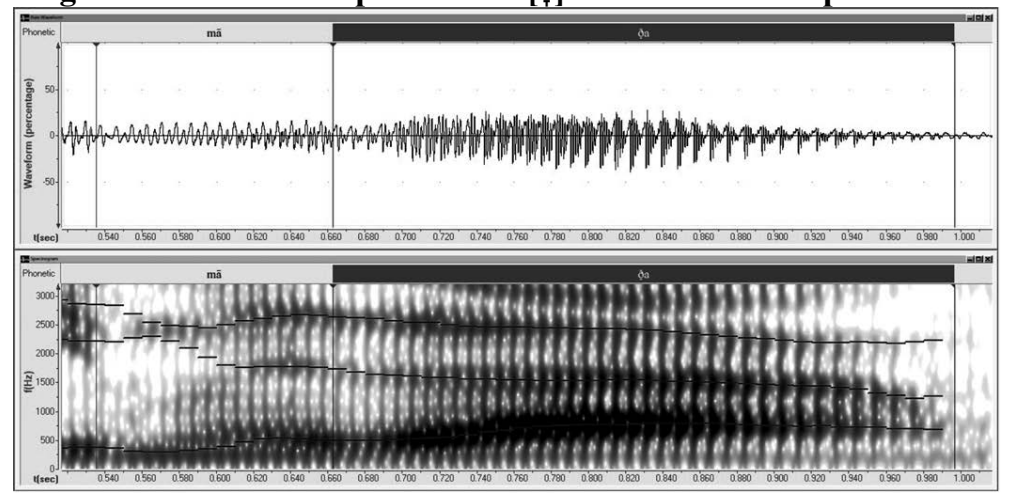

<madá> [mã.'ọa]

'también' 
Lo mismo ocurre con $/ \mathrm{g} /$; en la figura 6 se ejemplifica la realización fricativa $[\gamma]$ de este fonema. De la misma forma, no se registra la característica barra de oclusión en el eje vertical, sino una transición formántica continua como resultado de la fricativización:

Figura 6. Realización fricativa $[\gamma]$ del fonema /g/ en posición intervocálica

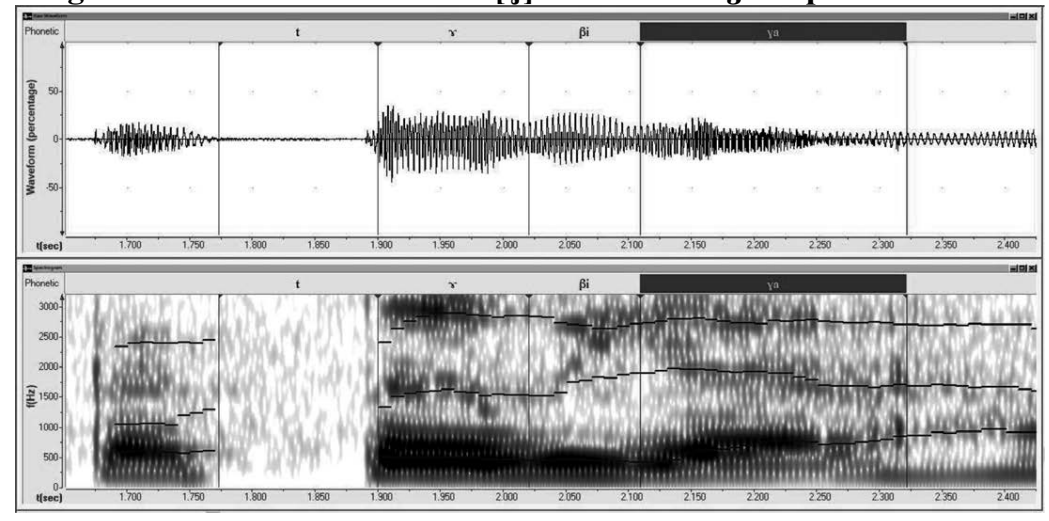

$<$ töbiga-bare> ['tr.ßi.ya] 'pensar-PRF'

En la figura 7, se muestra la manifestación aproximante []$^{32}$ de $/ \mathrm{g} /$. Los órganos articulatorios solo se aproximan sin establecer contacto, esto se refleja en la continuidad de la transición entre la aproximante y las vocales adyacentes:

32 Por simetría en la transcripción, se ha decidido transcribir el alófono aproximante de /g/ de la forma [ชู $[$, y no como aparece en Alfabeto Fonético Internacional ([u]]). 
Figura 7. Realización aproximante [?] del fonema /g/ en posición intervocálica

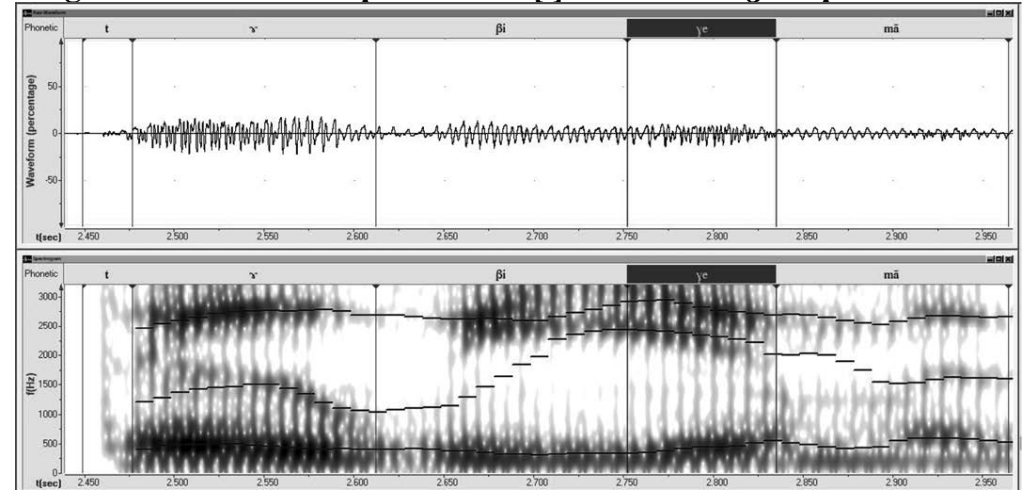

$<$ töbigué> [tr.ßi.'Ye]

'pensar-PRS'

\section{Elisión}

En cuanto a la elisión, los resultados obtenidos señalan que es poco frecuente en las oclusivas sordas. En las muestras de audio analizadas se encontraron solamente algunos casos marginales de elisión de la consonante velar sorda $/ \mathrm{k} /$ en entornos intervocálicos. En la figura 8 se muestra uno de estos casos:

\section{Figura 8. Elisión de /k/ en posición intervocálica}

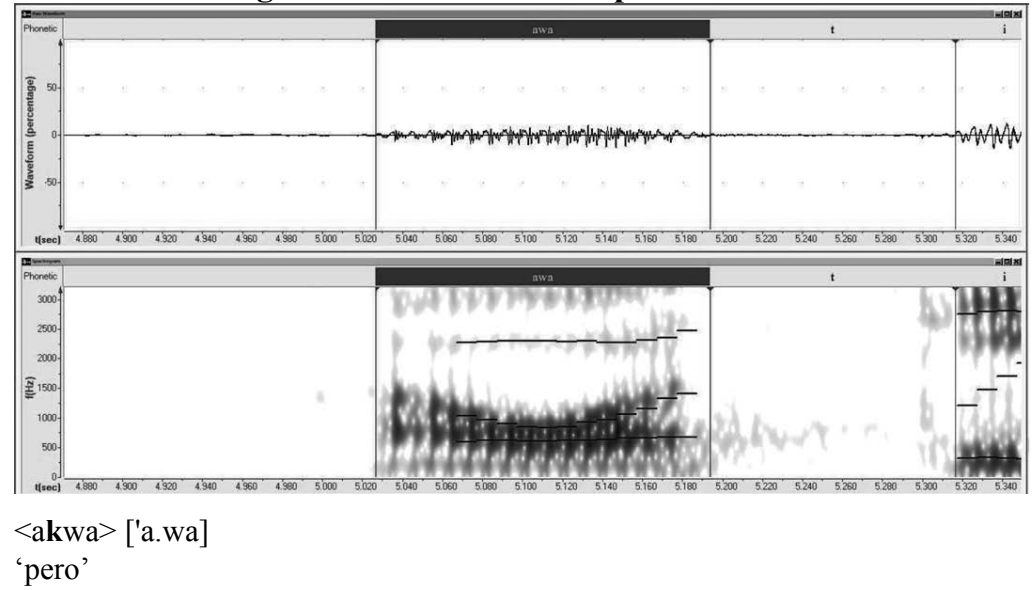


En las oclusivas sonoras la elisión es un proceso bastante frecuente como resultado del característico debilitamiento consonántico en entornos sonoros, generalmente intervocálicos. La oclusiva bilabial sonora presenta una tendencia mayor a la elisión en comparación con la dentoalveolar y velar. En la figura 9, un ejemplo de elisión del fonema $/ \mathrm{b} /$ :

Figura 9. Elisión de /b/ en posición intervocálica

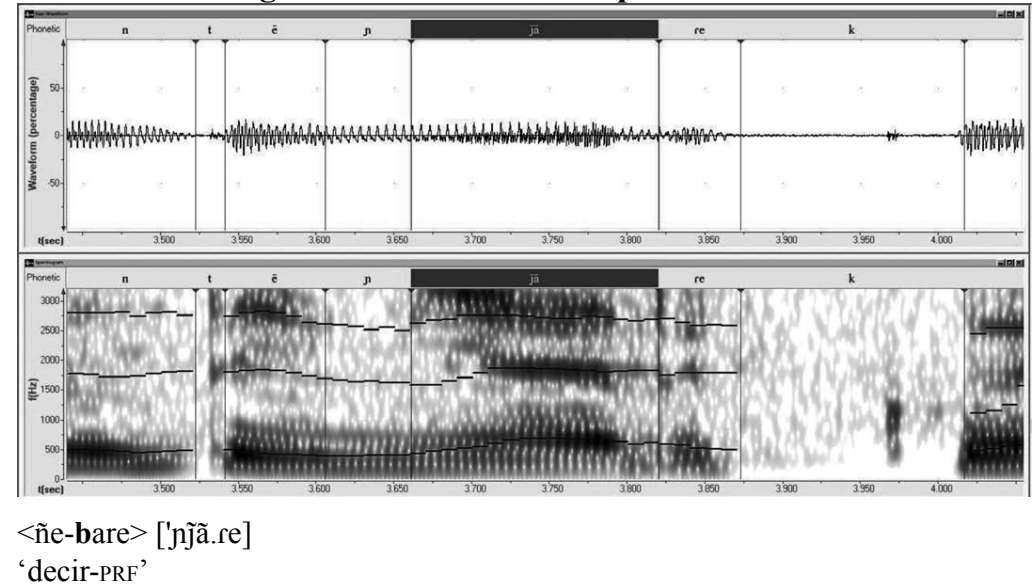

Es frecuente la caída del segmento silábico postónico a final de palabra en el que la bilabial ocupa la posición de ataque; esto se encuentra relacionado con procesos de debilitamiento que también afectan a las vocales. Al respecto, Juan Diego Quesada señala que en las lenguas chibchas de Centroamérica, además de la elisión vocálica, también es común la elisión de segmentos silábicos, generalmente postónicos, en palabras polisilábicas ${ }^{33}$. En la figura 10 se muestra la caída de un segmento silábico:

33 Juan Diego Quesada (2007), 54. 


\section{Figura 10. Caída de segmento silábico postónico}

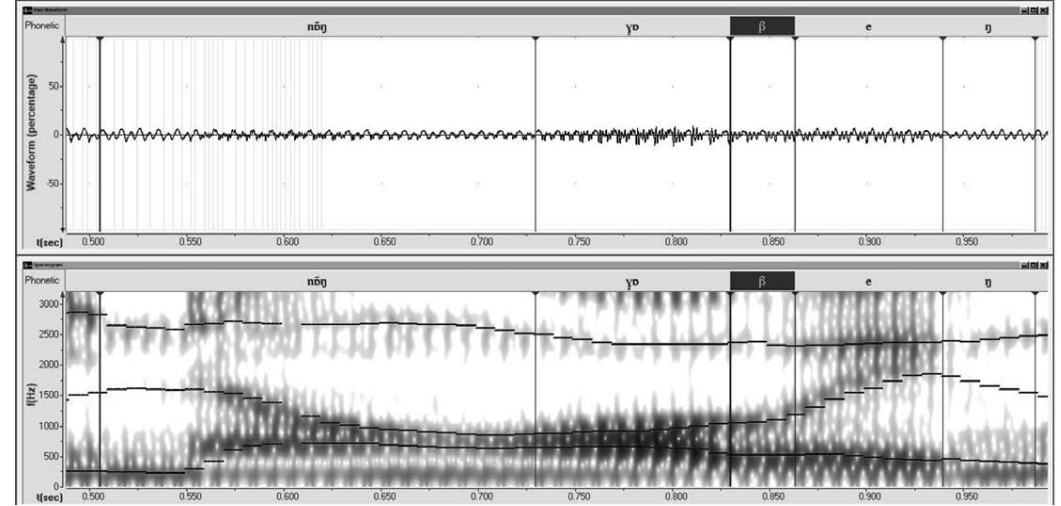

$<$ näm-ba ngäbe> [ñ̃y.' ' ’v.ßẽy]

'estar-AS gente'

En la figura 11 se presenta un ejemplo de elisión de la oclusiva dentoalveolar sonora. Como se muestra, no hay rastro de barra vertical de oclusión y la caída de la consonante resulta en un proceso de coalescencia o alargamiento vocálico.

Figura 11. Elisión de /d/ en posición intervocálica

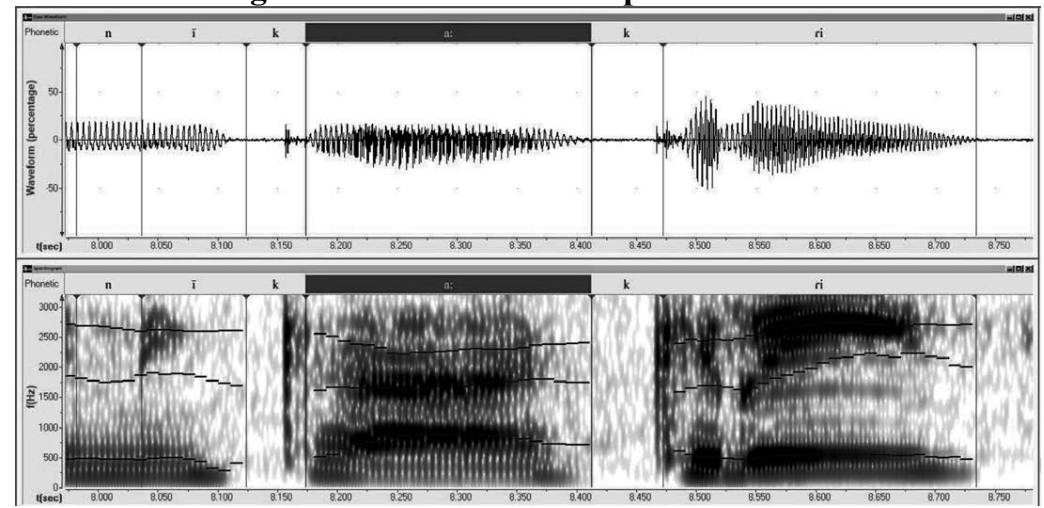

$<\mathrm{kada}>$ [ka:]

'boca' 
También es común la elisión de la consonante oclusiva velar sonora. En la figura 12, se representa la ausencia de la oclusiva se refleja en la continuidad de la transición formántica entre las vocales.

Figura 12. Elisión de /g/ en posición intervocálica

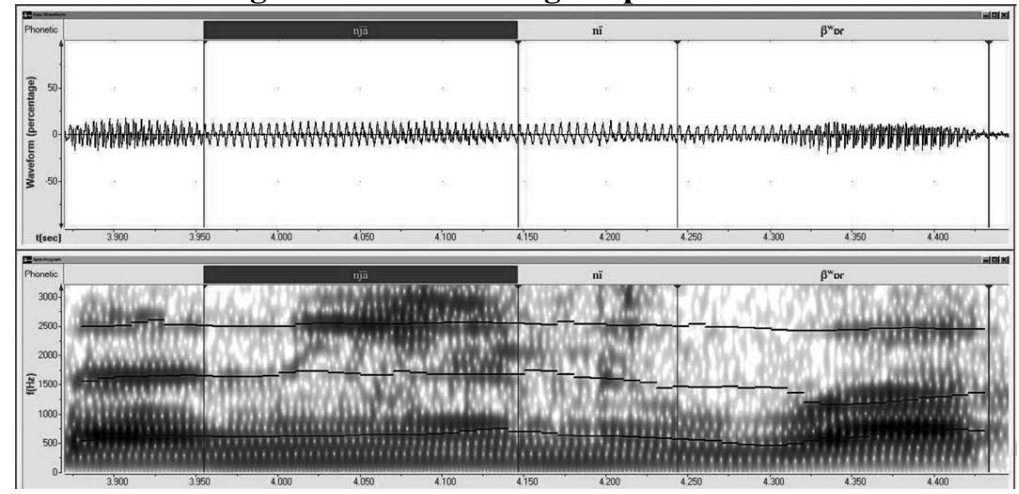

$<$ niga-ni> ['nj̃ã.nĩ]

'ir-P.REM'

\section{Asimilación}

En cuanto a los fenómenos de asimilación, es habitual la epéntesis de nasales homorgánicas por asimilación de las consonantes oclusivas. Incluso, en algunos casos son elementos remanentes que indican la elisión o caída de una consonante oclusiva. Como se verá en las muestras espectrográficas, los fonos nasales se caracterizan por contar con una nítida barra de sonoridad a lo largo de la zona inferior del eje horizontal y por concentraciones de armónicos a diferentes alturas.

En la muestra de la figura 13 se ve el caso del sonido nasal dentoalveolar sonoro [n] sugerido por el grafema $<\mathrm{n}>$ se articula como una nasal bilabial por efecto de la asimilación del punto de articulación de la oclusiva bilabial contigua: 
Figura 13. Articulación de nasal bilabial [m] por asimilación de [b]

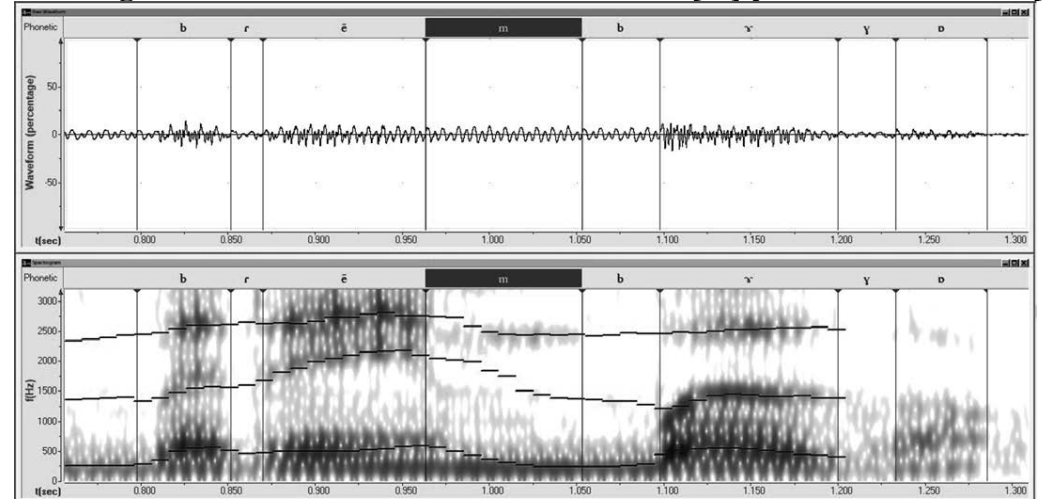

<bren bögän> [brẽm.'br.yp]

'enfermo demasiado'

La figura 14 presenta un caso de inserción de nasal dentoalveolar [n] como resultado de la asimilación por punto de articulación de la oclusiva dentoalveolar $[\mathrm{t}]$.

Figura 14. Epéntesis de nasal dentoalveolar [n] por asimilación de [t]

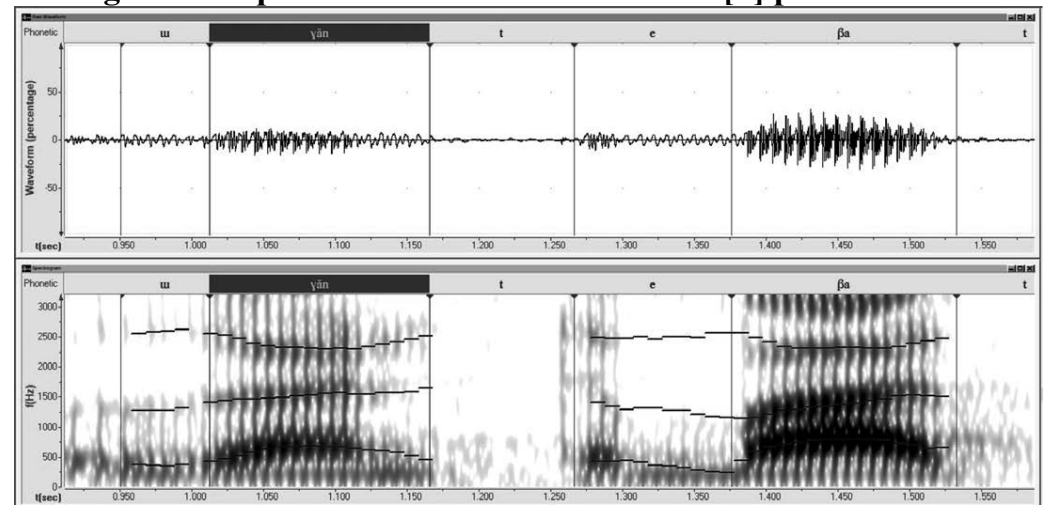

$<$ ügate-ba> [u.yãn.te.'ßa]

'arreglar-AS' 
En la figura 15 se muestra la inserción de la nasal velar [y] por asimilación al alófono fricativo velar $[\gamma]$ del fonema $/ \mathrm{g} /$.

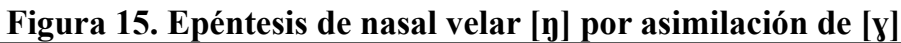

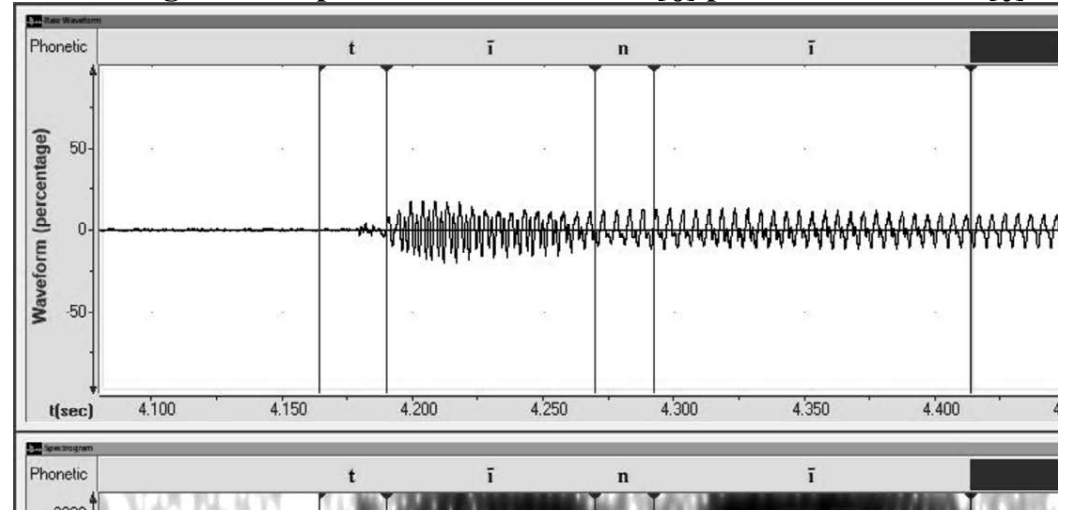

$<$ nigu-i> ['nĩn. $\cdot \gamma u]$

'ir-P.REC'

\section{Voice Onset Time}

El V.O.T. (Voice Onset Time) es la medida del tiempo que transcurre entre el inicio de la fase de explosión de una consonante oclusiva y el momento en que empieza la sonoridad. Fue propuesto por Lisker y Abramson ${ }^{34}$ como un rasgo acústico que en algunas lenguas permite diferenciar entre oclusivas sordas y sonoras, ya que, a pesar de que las series de oclusivas sordas y sonoras de muchas lenguas se distinguen por la presencia o ausencia de onda periódica, la pulsación glotálica no siempre es un rasgo suficiente para diferenciarlas ${ }^{35}$. En esta sección se determinarán los valores del V.O.T. de las consonantes oclusivas del guaymí, con el fin de conocer si este también puede ser un parámetro que se tome en cuenta para su diferenciación.

34 Leigh Lisker y Arthur S. Abramson, «A Cross-Language Study of Voicing in Initial Stops: Acoustical Measurements», Word 20 (1964): 384-422.

35 Yasna Roldán y Jaime Soto-Barba, «El V.O.T. de /p-t-k/ y /b-d-g/ en el español de Valdivia: un análisis acústico», Estudios filológicos 32 (1997): 27-33, §1. 
De la misma forma que Lisker y Abramson, en este análisis se le asigna al momento de la explosión el valor referencial cero, de modo que, cuando la sonoridad inicia después de la fase de explosión el V.O.T. adquiere valores positivos y, cuando inicia antes, negativos. Se analizó un total de 294 instancias de consonantes en posición inicial absoluta y en medio de palabra, con la ayuda de los cursores de tiempo de Speech Analyzer.

Como se muestra en la Tabla 1, los resultados muestran que el valor del V.O.T. de las consonantes oclusivas sordas es positivo en todos los casos analizados.

Tabla 1. Promedio del V.O.T. de las consonantes oclusivas sordas

\begin{tabular}{|l|c|}
\hline \multicolumn{1}{|c|}{ Oclusiva } & Promedio del V.O.T. (ms) \\
\hline Dentoalveolar $[\mathrm{t}]$ & 20.7 \\
\hline Velar $[\mathrm{k}]$ & 32.2 \\
\hline
\end{tabular}

En contraste, los resultados de la Tabla 2 muestran que el valor del V.O.T. de las consonantes oclusivas sonoras es negativo en todos los casos analizados, es decir, estas oclusivas se caracterizan por la presonoridad. El V.O.T. negativo se midió desde el inicio de la primera baja frecuencia periódica visible que indica el inicio de la sonoridad hasta la explosión de energía espectral que marca la liberación de energía; sin embargo, en algunos casos se observó ausencia de la barra de explosión.

Tabla 2. Promedio del V.O.T. de las consonantes oclusivas sonoras

\begin{tabular}{|l|c|}
\hline \multicolumn{1}{|c|}{ Oclusiva } & Promedio del V.O.T. (ms) \\
\hline Bilabial [b] & -66.6 \\
\hline Dentoalveolar [d] & -72.7 \\
\hline Velar [g] & -80.6 \\
\hline
\end{tabular}

Como se aprecia en la información de las tablas, el V.O.T. es mayor en las oclusivas sonoras. También, se identifica una estrecha 
relación entre el valor del V.O.T. y la zona de articulación, ya que, tanto en el caso de las sordas como en el de las sonoras, los valores aumentan a medida que el punto de articulación se vuelve posterior. Estos resultados muestran que, además de las vibraciones glotales, la duración del V.O.T. es un rasgo que contribuye a la distinción entre las oclusivas sordas y sonoras del guaymí.

\section{Conclusiones}

Sobre las oclusivas de la variedad costarricense del guaymí, este trabajo concluye lo siguiente:

- En primer lugar, con relación a lo expuesto por Murillo, se confirma que los fonemas oclusivos sordos $/ \mathrm{t} / \mathrm{y} / \mathrm{k} /$ no presentan variantes alofónicas sistemáticas. Las oclusivas sordas tienden a mantenerse incluso en entornos sonoros y, en contraste con lo mencionado por este autor, rara vez alternan con su correlato sonoro. Cabe decir que se registran algunos casos aislados en los que la consonante oclusiva velar sorda $/ \mathrm{k} /$ alterna con una realización fricativa velar sonora $[\mathrm{y}]$ como resultado del debilitamiento en entornos sonoros estrictamente vocálicos. Además, se corrobora que, de conformidad con lo planteado por Murillo, los fonemas oclusivos sonoros $/ \mathrm{b} /, / \mathrm{d} / \mathrm{y}$ $/ \mathrm{g} /$ presentan de forma sistemática alófonos oclusivos [b], [d]

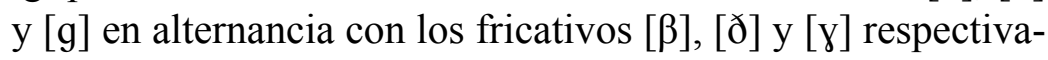
mente. Los primeros aparecen en posición inicial de palabra y,

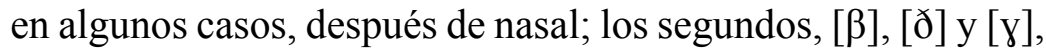
se articulan en entornos sonoros, generalmente vocálicos. En los datos analizados en esta investigación, además de los alófonos oclusivos y fricativos, también se registra la aparición de

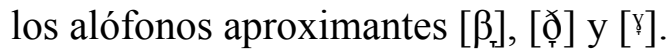

- En segundo lugar, con respecto a lo expuesto por Miguel Ángel Quesada Pacheco sobre la neutralización como un fenómeno 
común que afecta a los fonemas oclusivos sordos $/ \mathrm{t} / \mathrm{h} / \mathrm{k} / \mathrm{y}$ los sonoros $/ \mathrm{d} /, / \mathrm{g} /$ en la variedad panameña del guaymí, el presente análisis muestra que en el caso de la variedad estudiada, la alternancia entre oclusivas sordas y sonoras no ocurre con frecuencia; por el contrario, no se registraron alternancias entre las dentoalveolares y la oclusiva velar sorda presentó solamente alternancias aisladas con el fono fricativo velar [y]. En cuanto a las variaciones fricativas que presentan los fonemas oclusivos sonoros /b/, /d/ y /g/ debido a procesos de debilitamiento, se confirma que es un proceso que también ocurre en las oclusivas sonoras del guaymí de la variedad costarricense.

- En tercer lugar, con relación a lo señalado por Juan Diego Quesada acerca de procesos comunes de lenición consonántica en los sonidos obstruyentes de la familia chibcha, de las conclusiones anteriores se infiere que, por un lado, en el caso de la variedad del guaymí estudiada, la lenición ocurre de forma marginal en las consonantes oclusivas sordas, y por otro lado, como ocurre también en el buglere, los segmentos oclusivos sonoros tienden a la fricativización como resultado del debilitamiento en entornos con segmentos silábicos; sin embargo, en la variedad estudiada no se detecta una menor tendencia al debilitamiento en sílabas acentuadas. La lenición también puede resultar en casos de total elisión; sobre esto último, en el guaymí el fonema oclusivo bilabial sonoro es el que presenta una mayor tendencia.

- $\quad$ En cuarto y último lugar, el análisis del V.O.T. de las oclusivas de la variedad del guaymí hablado en Costa Rica muestra que este también es un parámetro que contribuye a la distinción entre oclusivas sordas y sonoras. Esto se debe a que el V.O.T. de las oclusivas sordas toma valores positivos, mientras que el de las sonoras, negativos y, además, es mayor. Sumado a esto, se identifica una estrecha relación entre el valor del V.O.T. y 
la zona de articulación, ya que tanto en el caso de las sordas como en el de las sonoras, los valores aumentan a medida que el punto de articulación se vuelve posterior.

Así pues, en este artículo se ha realizado una revisión de las descripciones más recientes del estatus fonológico de la serie oclusiva del guaymí hablado en Costa Rica; un análisis contrastivo entre la serie oclusiva del guaymí hablado en Costa Rica y la serie de la variedad panameña descrita por Miguel Ángel Quesada Pacheco; y una actualización sobre el estatus fonológico y las características acústicas de las oclusivas del guaymí de la variedad costarricense, mediante la descripción de procesos fonológicos y el análisis de los valores del V.O.T.

Finalmente, a modo de recomendación, se aconseja extender este estudio a otros poblados guaymíes del territorio costarricense y, por tanto, a otras posibles variedades del guaymí hablado en Costa Rica, con el fin de detectar posibles diferencias del estatus fonológico de las oclusivas y contar con un registro más amplio del comportamiento de la serie oclusiva en la lengua. Además, para obtener diferencias acústicas más detalladas entre oclusivas sordas y sonoras, se recomienda profundizar en el análisis de los aspectos del contexto fonético-fonológico que pueden estar asociados a los valores del V.O.T.

\section{Lista de abreviaturas de la traducción morfemática}

as

dem

p. rec

p. rem

prf

prs

refl

sg asertivo

demostrativo

pasado reciente

pasado remoto

perfecto

presente

reflexivo

singular 